\title{
Recurrence rate after pressure ulcer reconstruction in patients with spinal cord injury in patients under control by a plastic surgery and physical medicine and rehabilitation team
}

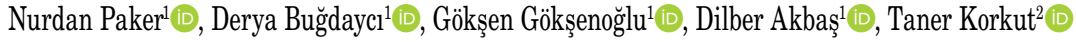 \\ ${ }^{1}$ Department of Physical Medicine and Rehabilitation, İstanbul Physical Therapy and Rehabilitation Training and Research Hospital, İstanbul, Turkey \\ ${ }^{2}$ Department of Plastic, Reconstructive and Aesthetic Surgery, Private Elite Polyclinic, İstanbul, Turkey
}

Received: October 04, 2017 Accepted: January 20, 2018 Published online: November 05, 2018

\begin{abstract}
Objectives: This study aims to evaluate the recurrence rate after reconstruction of the pressure ulcer (PU) in patients with spinal cord injury (SCI) treated by a team consisted of plastic surgery and physical medicine and rehabilitation specialists.

Patients and methods: Between February 2002 and December 2008, a total of 39 patients with SCI (32 males, 7 females; mean age $38.1 \pm 6.7$ years; range, 19 to 71 years) aged $\geq 18$ years who were admitted to our rehabilitation unit and operated for PU were retrospectively analyzed. Demographic data and physical examination findings and potential risk factors for recurrence of PU were recorded. All patients were followed for the PU recurrence after reconstruction.

Results: The recurrence of PU was observed in nine patients (23.1\%) with SCI. The most common recurrent PU was sacral ulcer (82.1\%), followed by ischial ulcers (20.5\%), trochanteric ulcers (15.4\%), and others (2.6\%). No risk factor was found to be statistically significant between the two groups in terms of both demographic risk factors and clinical risk factors. No statistically significant effect of clinical and demographic risk factors was found on the risk of recurrence of PU.

Conclusion: Our study results suggest that collaboration between plastic surgery and physical medicine and rehabilitation department with a strict follow-up protocol may help to prevent recurrences of PU after surgery in SCI patients.
\end{abstract}

Keywords: Operative; pressure ulcer; recurrence; spinal cord injury; surgical procedures.

Pressure ulcer $(\mathrm{PU})$ is one of the major complications which can be seen in patients with spinal cord injury (SCI). In particular, in chronic SCI with loss of sensation and comorbidity, PU is seen more frequently. ${ }^{[1]}$ The PU prevalence may increase up to 30 to $50 \%$ in SCI cases with increased morbidity and mortality. ${ }^{[2-5]}$ Pressure ulcers can be seen in $36.5 \%$ of the patients in the acute rehabilitation period and in $39.4 \%$ of the patients in the functional rehabilitation period. ${ }^{[6]}$ It typically involves sacrum, heel, and ischium in $43 \%, 19 \%$, and $15 \%$ cases, respectively. ${ }^{[6]}$ The incidence of PU has been reported as $26.4 \%$ in wheelchair patients with chronic SCI. In addition, almost one-third of these patients have previous PU before, and nearly half of them are newly diagnosed PU cases. ${ }^{[7]}$
Prevention of PU is of utmost importance for patients with SCI. After PU occurs, proper positioning to decrease the pressure is helpful for healing in Stage I and II ulcers. In the patients with Stage III-IV PU, surgery is usually indicated. However, healing in good quality is mostly difficult due to the lack of adequate soft tissues to fill the gap in deep ulcers. ${ }^{[8]}$ For patients whose PU is unable to recover properly with conservative treatment or those who have Stage III-IV $\mathrm{PU}$ wishing a rapid recovery, plastic surgery consultation is recommended. ${ }^{[9]}$ Various techniques can be used in the surgical treatment of PU, ${ }^{[10-14]}$ mainly including musculocutaneous, fasciocutaneous, and perforatorbased flaps. These flaps used in PU reconstruction have similar complication and recurrence rates. ${ }^{[11,12]}$

Corresponding author: Gökşen Gökşenoğlu, MD. İstanbul Fizik Tedavi ve Rehabilitasyon Eğitim ve Araştırma Hastanesi, Fizik Tedavi ve Rehabilitasyon Kliniği, 34186 Bahçelievler, İstanbul, Turkey. e-mail: goksengoksenoglu@hotmail.com 
In the present study, we aimed to investigate the recurrence rates in patients with SCI who had PU reconstruction and were followed by both by plastic surgery and physical medicine and rehabilitation team.

\section{PATIENTS AND METHODS}

Between February 2002 and December 2008, a total of 39 patients with SCI ( 32 males, 7 females; mean age $38.1 \pm 6.7$ years; range, 19 to 71 years) aged $\geq 18$ years who were admitted to our rehabilitation unit and operated for PU were retrospectively analyzed. Physical examination findings and accompanying disorders were recorded. The affected body sites were noted. Potential risk factors for PU recurrence such as anemia, smoking, and hypoalbuminemia were evaluated. A written informed consent was obtained from each patient. The study protocol was approved by the İstanbul Physical Medicine and Rehabilitation Training and Research Hospital local Ethics Committee. The study was conducted in accordance with the principles of the Declaration of Helsinki.

In 10 patients, more than one PU were treated surgically. A total of $39 \mathrm{PU}$ reconstructions were done in patients with SCI. For the surgical treatment of sacral PU, the first debridement was done and, then, the ulcer was closed with a random pattern fasciocutaneous rotation flap. Trochanteric pressure ulcers were closed with a random pattern transposition flap following the debridement. For the surgical treatment of ischial PU, the first debridement was done and, then, the deep part of the ulcer was closed with a muscle flap and the upper part was closed using the primary suture. For the surgical treatment of other PU cases, the primary suture, skin graft, or fasciocutaneous flaps were used. While choosing the proper flap for the reconstruction of PU, closing the ulcer easily was considered. Furthermore, it was considered that leaving potential flap in case of recurrence was essential in patients with SCI, as PU recurrence is common in these patients. For a three-week period following the operation, the patients were laid in the proper position to prevent any pressure, shearing or friction forces on the operated region, not to disturb the blood flow of the flap. The patients who had trochanteric PU reconstruction were laid in the lateral decubitus position, while those who had sacral PU reconstruction were laid in the prone position. For patients lying in the prone position, one pillow was put under the head, two pillows were used at chest, and one pillow was used to support the pelvic area and legs. For this three-week bed rest period, clean intermittent catheterization was not applied.
During this period, continuous catheterization was used for urine discharge in all patients. In particular, postoperative sacral PU has the risk of fecal contamination, as they are close to the anal region, and toilet care in these patients is very important. Accordingly, all patients had a regular diet containing enough water and fiber for making the stool bulkier in the postoperative period. Moreover, an anal pad was used during defecation to keep the wound clean in this period. Wound dressing was performed for ulcer. Drainage, flap color, and edema were monitored regularly. Drains were removed weekly. The stitches were removed at the second week after the operation. After completing the three-week bed rest period, the patients were included in the rehabilitation program, unless they had any problem related with ulcers.

All patients with PU reconstruction were followed in our inpatient rehabilitation unit postoperatively. The caregivers were continuously trained for preventing the ulcer recurrence. All patients were informed in detail about the skin care and pressurelowering methods.

Rehabilitation program consisted of slowly progressive exercises according to the health status of the patient. Also, pressure relief exercises were instructed to all patients. All patients were also followed for PU after discharge.

\section{Statistical analysis}

Statistical analysis was performed using the PASW Statistics, version 18.0 software (SPSS Inc., Chicago, IL, USA). Descriptive data were expressed in mean and standard deviation (SD) and median (min-max), and number and percent for categorical variables. All data were analyzed for normality of distribution using the Kolmogorov-Smirnov test with the Lilliefors Significance Correction. Comparisons of the two groups in terms of age, body height, weight,

Table 1. Demographic and anthropometric characteristics

\begin{tabular}{lcccc}
\hline Recurrence & Age & Weight & Height & BMI \\
\hline No $(\mathrm{n}=30)$ & & & & \\
$\quad$ Median & 29.5 & 67.5 & 170.0 & 23.1 \\
$\quad$ Minimum & 19.0 & 40.0 & 158.0 & 16.0 \\
$\quad$ Maximum & 71.0 & 85.0 & 188.0 & 27.7 \\
Yes (n=9) & & & & \\
$\quad$ Median & 32.0 & 65.0 & 168.0 & 23.9 \\
$\quad$ Minimum & 21.0 & 49.0 & 157.0 & 18.7 \\
$\quad$ Maximum & 56.0 & 82.0 & 183.0 & 27.7 \\
P value & 0.960 & 0.853 & 0.258 & 0.423 \\
\hline
\end{tabular}

BMI: Body Mass Index. 
Table 2. Patients distribution as frequencies, percentages and Odds ratios for the potential demographic risk factors

\begin{tabular}{|c|c|c|c|c|c|c|c|c|}
\hline & \multicolumn{4}{|c|}{ Recurrence } & \multirow[b]{3}{*}{$\mathrm{P}$ value } & \multicolumn{3}{|c|}{ Odds ratio } \\
\hline & \multicolumn{2}{|c|}{ No } & \multicolumn{2}{|c|}{ Yes } & & \multirow[b]{2}{*}{ Value } & \multirow[b]{2}{*}{ Lower limit } & \multirow[b]{2}{*}{ Upper limit } \\
\hline & $\mathrm{n}$ & $\%$ & $\mathrm{n}$ & $\%$ & & & & \\
\hline \multicolumn{9}{|l|}{ Gender } \\
\hline Female & 6 & 20 & 1 & 11.1 & \multirow{2}{*}{1.000} & \multirow{2}{*}{2.000} & \multirow{2}{*}{0.208} & \multirow{2}{*}{19.227} \\
\hline Male & 24 & 80 & 8 & 88.9 & & & & \\
\hline \multicolumn{9}{|l|}{ Marital status } \\
\hline Single & 16 & 53.3 & 5 & 55.6 & \multirow{2}{*}{1.000} & \multirow{2}{*}{0.914} & \multirow{2}{*}{0.204} & \multirow{2}{*}{4.088} \\
\hline Married & 14 & 46.7 & 4 & 44.4 & & & & \\
\hline \multicolumn{9}{|l|}{ Smoking } \\
\hline No & 23 & 76.7 & 5 & 55.6 & \multirow{2}{*}{0.238} & \multirow{2}{*}{2.629} & \multirow{2}{*}{0.550} & \multirow{2}{*}{12.552} \\
\hline Yes & 7 & 23.3 & 4 & 44.4 & & & & \\
\hline \multicolumn{9}{|l|}{ Education } \\
\hline Primary school & 22 & 73.3 & 9 & 100 & 0.160 & 0.710 & 0.567 & 0.889 \\
\hline High school & 8 & 26.7 & 0 & 0 & & & & \\
\hline
\end{tabular}

Body Mass Index (BMI), time since injury, length of stay, length of follow-up were performed using the Mann-Whitney U test. In terms of the potential risk factors, patient distribution between two groups was analyzed using the Fisher's exact test. The odds ratios (ORs) with 95\% confidence interval (CI) for potential risk factors were calculated. A $p$ value of $<0.05$ was considered statistically significant.

\section{Sample size and post-hoc power analysis}

The primary aim of this study was to evaluate the recurrence rate after reconstruction of the PU. In the literature, the rate of postoperative recurrence of $\mathrm{PU}$ in SCI patients has been reported as $8.9 \% .{ }^{[10]}$ Therefore, a total of 26 patients are needed to have $80 \%$ power with $5 \%$ type I error level to detect effect size $(-0.168)$ to achieve an expected rate of postoperative recurrence of $8.9 \%$. The post-hoc power $(n=39$, effect size $=-0.168$, $\alpha=0.05$ (two-tail) is 0.993 ( $\mathrm{G}^{*}$ power ver 3.1.9.2 Franz Paul Germany).

\section{RESULTS}

Demographic and clinical characteristics of the patients are shown in Tables 1 and 2. The PU recurrence rate was nine (23.1\%). The most common recurrent PU was sacral ulcers $(82.1 \%)$, followed by ischial ulcers (20.5\%), trochanteric ulcers (15.4\%), and others (2.6\%). The PU recurrence was seen in two sites in six patients and three sites in one patient. Anatomical localizations of recurrent Pus are shown in Figure 1.

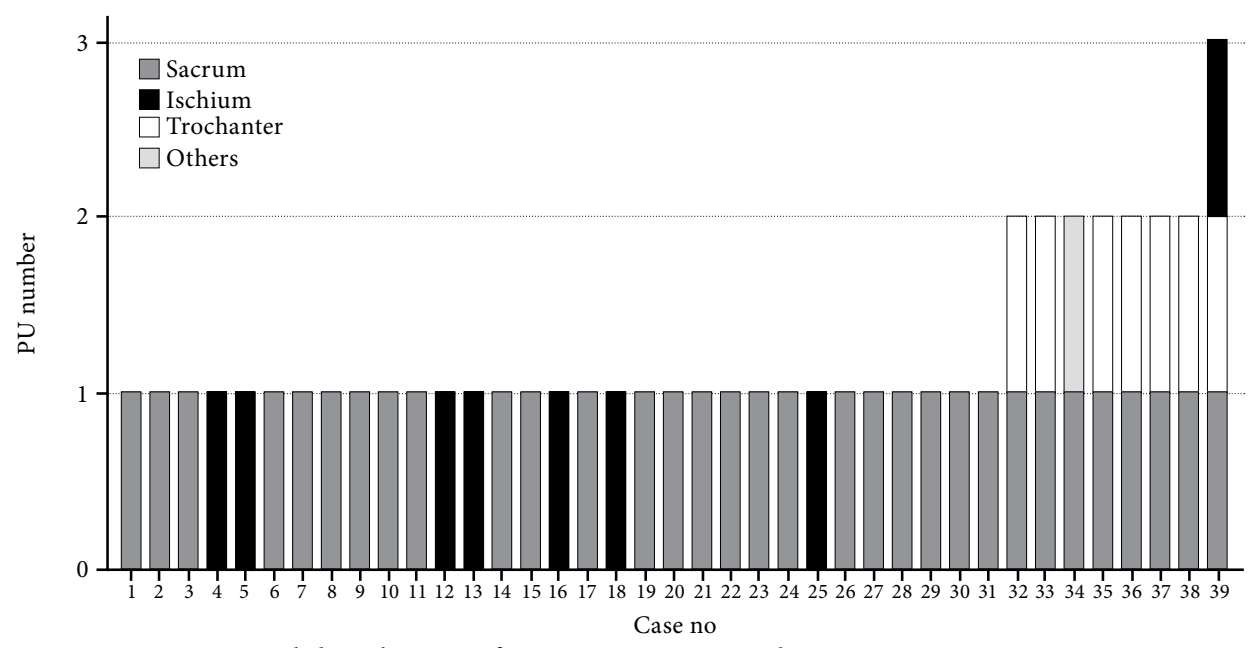

Figure 1. Anatomical distribution of recurrent pressure ulcers. PU: Pressure ulcer. 
Table 3. Patients distribution as frequencies, percentages and Odds ratios for the potential clinical risk factors

\begin{tabular}{|c|c|c|c|c|c|c|c|c|}
\hline & \multicolumn{4}{|c|}{ Recurrence } & \multirow[b]{3}{*}{$\mathrm{P}$ value } & \multicolumn{3}{|c|}{ Odds ratio } \\
\hline & \multicolumn{2}{|c|}{ No } & \multicolumn{2}{|c|}{ Yes } & & \multirow[b]{2}{*}{ Value } & \multirow[b]{2}{*}{ Lower limit } & \multirow[b]{2}{*}{ Upper limit } \\
\hline & $\mathrm{n}$ & $\%$ & $\mathrm{n}$ & $\%$ & & & & \\
\hline \multicolumn{9}{|l|}{ Injury } \\
\hline Complete & 17 & 56.7 & 6 & 66.7 & \multirow{2}{*}{0.711} & \multirow{2}{*}{0.654} & \multirow{2}{*}{0.137} & \multirow{2}{*}{3.120} \\
\hline Incomplete & 13 & 43.3 & 3 & 33.3 & & & & \\
\hline \multicolumn{9}{|l|}{ Etiology } \\
\hline Traumatic & 18 & 60 & 7 & 77.8 & \multirow{2}{*}{0.445} & \multirow{2}{*}{0.429} & \multirow{2}{*}{0.076} & \multirow{2}{*}{2.425} \\
\hline Violence & 12 & 40 & 2 & 22.2 & & & & \\
\hline \multicolumn{9}{|l|}{ Exercise } \\
\hline No & 10 & 33.3 & 6 & 66.7 & \multirow{2}{*}{0.123} & \multirow{2}{*}{0.250} & 0051 & 1214 \\
\hline Yes & 20 & 66.7 & 3 & 33.3 & & & 0.031 & 1.214 \\
\hline Spasticity & & & & & & & & \\
\hline No & 16 & 53.3 & 7 & 77.8 & 0262 & 0327 & 0058 & \\
\hline Yes & 14 & 46.7 & 2 & 22.2 & 0.262 & 0.321 & 0.058 & $1.83 /$ \\
\hline Voiding & & & & & & & & \\
\hline Spontaneous & 4 & 13.3 & 2 & 22.2 & 0.607 & 0.538 & 0.081 & 3.569 \\
\hline Catheterization & 26 & 86.6 & 7 & 77.8 & & & & \\
\hline Pain & & & & & & & & \\
\hline No & 20 & 66.7 & 7 & 77.8 & 0963 & 0.571 & 0100 & 3273 \\
\hline Yes & 10 & 33.3 & 2 & 22.2 & 0.700 & 0.071 & 0.100 & 0.270 \\
\hline Infection & & & & & & & & \\
\hline No & 13 & 43.3 & 4 & 44.4 & 1000 & 0956 & 0213 & 4284 \\
\hline Yes & 17 & 56.7 & 5 & 55.6 & 1.000 & 0.950 & 0.213 & 4.204 \\
\hline Ambulation & & & & & & & & \\
\hline Wheelchair & 10 & 33.3 & 3 & 33.3 & 1000 & 1000 & 0206 & 4856 \\
\hline Ambulatory & 20 & 66.7 & 6 & 66.6 & 1.000 & 1.000 & 0.200 & 4.850 \\
\hline DVT & & & & & & & & \\
\hline No & 28 & 93.3 & 8 & 88.9 & 0.556 & 1.750 & 0.140 & 21.876 \\
\hline Yes & 2 & 6.7 & 1 & 11.1 & & & & \\
\hline $\mathrm{HO}$ & & & & & & & & \\
\hline No & 27 & 90 & 8 & 88.9 & 1000 & 1125 & 0102 & 12361 \\
\hline Yes & 3 & 10 & 1 & 11.1 & 1.000 & $1.12 J$ & 0.102 & 12.001 \\
\hline Fracture & & & & & & & & \\
\hline No & 21 & 70 & 5 & 55.6 & 0.447 & 1867 & & \\
\hline Yes & 9 & 30 & 4 & 44.4 & $0.44 /$ & $1.86 /$ & 0.405 & 8.614 \\
\hline Anemia & & & & & & & & \\
\hline No & 12 & 40 & 2 & 22.2 & 0.445 & 2.333 & 0.412 & 13.200 \\
\hline Yes & 18 & 60 & 7 & 77.8 & & & & \\
\hline Hypoalbuminemia & & & & & & & & \\
\hline No & 23 & 76.7 & 5 & 55.6 & 0238 & 2629 & 0.550 & 12552 \\
\hline Yes & 7 & 23.3 & 4 & 44.4 & 0.250 & 2.023 & 0.030 & 12.032 \\
\hline Multipl operation & & & & & & & & \\
\hline No & 22 & 73.3 & 7 & 77.8 & 1000 & 0786 & 0134 & 4602 \\
\hline Yes & 8 & 26.7 & 2 & 22.2 & 1.000 & 0.180 & 0.134 & 4.002 \\
\hline Reoperation & & & & & & & & \\
\hline No & 25 & 83.3 & 7 & 77.8 & & & & \\
\hline Yes & 5 & 16.7 & 2 & 22.2 & 0.653 & 1.429 & 0.227 & 9.009 \\
\hline
\end{tabular}

The mean length of stay was 80.5 (range, 21 to 167 ) days in the patients without recurrence and 106 (range, 22 to 197) days in the patients with recurrence $(\mathrm{p}=0.334)$. The mean time since injury was 29.5 (range, 12 to 196) months and 36 (range, 16 to 288 ) months, respectively $(\mathrm{p}=0.515)$. The mean follow-up 
was 20.5 (range, 6 to 33) months in the patients without recurrence and 21 (range, 6 to 26) months in the patients with recurrence $(\mathrm{p}=0.413)$.

No risk factor was found to be statistically significant between the two groups in terms of both demographic risk factors (Table 2) and clinical risk factors (Table 3). No statistically significant effect of clinical and demographic risk factors was found on the recurrence risk of PU (Tables 2 and 3).

\section{DISCUSSION}

In this study, the recurrence rate was $23.1 \%$ in patients with SCI who had PU reconstruction during a mean follow-up of $19.8 \pm 7.5$ months. Almost one-fifth of the patients who had PU recurrence were those who were already operated for previous ulcers in external medical centers. The patients who had previous recurrence were also at risk of increased PU after a new operation.

The recurrence rate after reconstruction of the PU can be as high as 47.8 to $56 \%$ among the patients with SCI. ${ }^{[14,15]}$ In a study by Kierney et al., ${ }^{[16]}$ a team work was carried out including the plastic surgery department for surgical initiative and the physical medicine and rehabilitation department for follow-up, and the recurrence rate was found to be $19.5 \%$ in 268 patients at a mean age of 34.5 years and who were operated for Stage III and IV PU and followed for a mean period of 3.7 years. In the aforementioned study, the authors concluded that the low recurrence rate in the surgical treatment of PU was the result of the team work between the plastic surgery and physical medicine and rehabilitation departments. In our study, the recurrence rates are similar to the aforementioned study, and the whole treatment process was carried out by a team of physicians and nurses of physical medicine and rehabilitation department and a plastic surgeon. A multidisciplinary team work is, hence, required for the treatment of PU. Rehabilitation specialists and nurses have important responsibilities in the PU treatment with the plastic surgery specialists.

Pressure relief is also helpful for preventing ulcer in the regions that are exposed to pressure for a long time. ${ }^{[4]}$ It is recommended that pressure-lowering maneuvers should be repeated for half to two min with 15 to 30 -min intervals. ${ }^{[17]}$ For patients with SCI, prolonged sitting is another risk factor in terms of ischial PU. ${ }^{[18]}$ For those confined to wheelchair ambulation, regular exercises should be done and pressure relief positions should be ensured. Leaning forward also helps lowering pressure in the ischial region for patients who are sitting on a wheelchair. ${ }^{[19]}$ In a study carried out by Rintala et al. ${ }^{[20]}$ including patients with SCI and PU, the PU frequency could be decreased in the group followed by phone calls following the PU education on a monthly basis, compared to those who were only followed by phone calls or emails without any education.

In the present study, sacral PU was closed by randomly patterned rotation flap. No postoperative flap necrosis was observed in any patients. In a study by Chen et al., ${ }^{[21]}$ the gluteal rotator flap and fasciocutaneous rotation flap applications were compared in patients with sacral PU and recurrence was seen in patients in whom a fasciocutaneous rotation flap was used. However, the authors concluded that both techniques were effective in the treatment of sacral PU. Sørensen et al..$^{[8]}$ also reported that myocutaneous flaps were mostly used in the patients who underwent PU reconstruction. In another study carried out by Leow et al., ${ }^{[22]}$ four patients with paraplegia or traumatic brain injury were evaluated and sacral PU reconstructions with superior gluteal perforator flaps were found to be successful and the gluteal muscles were reserved in case of recurrence as they remained intact. The authors also found that recurrence could be observed after $\mathrm{PU}$ reconstruction and, in this case, it was of utmost importance to leave the potential usable flaps for the future surgical interventions.

In our study, the majority of the operated Pus were in the sacrum region. Bardak et al..$^{[5]}$ showed that sacral region was the most commonly affected site in $44.4 \%$ of cases with SCI. Sacral PU is most common in in-patients. Therefore, these patients should be supported in terms of skin care precautions, position changes, and proper exercises which can lower pressure on the sacral region.

This study has some strengths. First, it has a long follow-up period for recurrence in patients with SCI with a previous PU reconstruction. Second, all $\mathrm{PU}$ reconstructions were done in a single center. Another strength is the multidisciplinary work of plastic surgery specialists and physical medicine and rehabilitation team during the whole process before and after the surgical treatment. Nonetheless, the lack of a logistic regression analysis to identify risk factors of PU and confounding effects among potential risk factors is the main limitation of this study. In addition, log-linear analysis which examines the relationship between more than two categorical variables was unable to be performed to identify PU-related risk 
factors, due to small sample size. Therefore, further large-scale, prospective studies are needed to fully delineate possible risk factors of PU recurrence.

In conclusion, our study results suggest that collaboration between plastic surgery and physical medicine and rehabilitation department with a strict follow-up protocol may help to prevent recurrences of PU after surgery in SCI patients. In addition, regular pressure relief exercises following surgery are associated with a lower recurrence risk in these patients.

\section{Declaration of conflicting interests}

The authors declared no conflicts of interest with respect to the authorship and/or publication of this article.

\section{Funding}

The authors received no financial support for the research and/or authorship of this article.

\section{REFERENCES}

1. Kruger EA, Pires M, Ngann Y, Sterling M, Rubayi S. Comprehensive management of pressure ulcers in spinal cord injury: current concepts and future trends. J Spinal Cord Med 2013;36:572-85.

2. Gélis A, Dupeyron A, Legros P, Benaïm C, Pelissier J, Fattal C. Pressure ulcer risk factors in persons with spinal cord injury part 2: the chronic stage. Spinal Cord 2009;47:651-61.

3. Byrne DW, Salzberg CA. Major risk factors for pressure ulcers in the spinal cord disabled: a literature review. Spinal Cord 1996;34:255-63.

4. Krause JS. Skin sores after spinal cord injury: relationship to life adjustment. Spinal Cord 1998;36:51-6.

5. Bardak AN, Erhan B, Gündüz B, Seyyah Ö, Boriçi S. Prevalance and risk factors of pressure ulcers in spinal cord injured patients. Turk J Phys Med Rehab 2010;56:26-9.

6. Verschueren JH, Post MW, de Groot S, van der Woude LH, van Asbeck FW, Rol M. Occurrence and predictors of pressure ulcers during primary in-patient spinal cord injury rehabilitation. Spinal Cord 2011;49:106-12.

7. Kovindha A, Kammuang-Lue P, Prakongsai P, Wongphan T. Prevalence of pressure ulcers in Thai wheelchair users with chronic spinal cord injuries. Spinal Cord 2015;53:767-71.

8. Sørensen JL, Jørgensen B, Gottrup F. Surgical treatment of pressure ulcers. Am J Surg 2004;188:42-51.

9. National Pressure Ulcer Advisory Panel, European Pressure Ulcer Advisory Panel and Pan Pacific Pressure Injury Alliance. Prevention and Treatment of Pressure Ulcers: Quick Reference Guide. In: Haesler E, editör. Osborne Park: Cambridge Media; 2014.
10. Yamamoto Y, Tsutsumida A, Murazumi M, Sugihara T. Long-term outcome of pressure sores treated with flap coverage. Plast Reconstr Surg 1997;100:1212-7.

11. Sameem M, Au M, Wood T, Farrokhyar F, Mahoney J. A systematic review of complication and recurrence rates of musculocutaneous, fasciocutaneous, and perforator-based flaps for treatment of pressure sores. Plast Reconstr Surg 2012;130:67-77.

12. Kuo PJ, Chew KY, Kuo YR, Lin PY. Comparison of outcomes of pressure sore reconstructions among perforator flaps, perforator-based rotation fasciocutaneous flaps, and musculocutaneous flaps. Microsurgery 2014;34:547-53.

13. Sapountzis S, Park HJ, Kim JH, Chantes A, Beak RM, Heo CY. The 'reading man flap' for pressure sore reconstruction. Indian J Plast Surg 2011;44:448-52.

14. Tavakoli K, Rutkowski S, Cope C, Hassall M, Barnett R, Richards $M$, et al. Recurrence rates of ischial sores in paraand tetraplegics treated with hamstring flaps: an 8-year study. Br J Plast Surg 1999;52:476-9.

15. Relander M, Palmer B. Recurrence of surgically treated pressure sores. Scand J Plast Reconstr Surg Hand Surg 1988;22:89-92.

16. Kierney PC, Engrav LH, Isik FF, Esselman PC, Cardenas DD, Rand RP. Results of 268 pressure sores in 158 patients managed jointly by plastic surgery and rehabilitation medicine. Plast Reconstr Surg 1998;102:765-72.

17. Consortium for Spinal Cord Medicine Clinical Practice Guidelines. Pressure ulcer prevention and treatment following spinal cord injury: a clinical practice guideline for health-care professionals. J Spinal Cord Med 2001;24 Suppl 1:S40-101.

18. Guihan M, Garber SL, Bombardier CH, Goldstein B, Holmes SA, Cao L. Predictors of pressure ulcer recurrence in veterans with spinal cord injury. J Spinal Cord Med 2008;31:551-9.

19. Regan MA, Teasell RW, Wolfe DL, Keast D, Mortenson WB, Aubut JA. A systematic review of therapeutic interventions for pressure ulcers after spinal cord injury. Arch Phys Med Rehabil 2009;90:213-31.

20. Rintala DH, Garber SL, Friedman JD, Holmes SA. Preventing recurrent pressure ulcers in veterans with spinal cord injury: impact of a structured education and follow-up intervention. Arch Phys Med Rehabil 2008;89:1429-41.

21. Chen YC, Huang EY, Lin PY. Comparison of gluteal perforator flaps and gluteal fasciocutaneous rotation flaps for reconstruction of sacral pressure sores. J Plast Reconstr Aesthet Surg 2014;67:377-82.

22. Leow M, Lim J, Lim TC. The superior gluteal artery perforator flap for the closure of sacral sores. Singapore Med J 2004;45:37-9. 